\title{
Biomassa seca acumulada, partições e rendimento industrial da cana-de-açúcar irrigada no Semiárido brasileiro ${ }^{1}$
}

\author{
Thieres George Freire da Silva', Magna Soelma Beserra de Moura ${ }^{3}$, Sérgio Zolnier \\ Luciana Sandra Bastos de Souza ${ }^{5}$ \\ http://dx.doi.org/10.1590/0034-737X201461050012
}

\begin{abstract}
RESUMO
Objetivou-se, com este trabalho, determinar parâmetros de crescimento e de rendimento da cana-de-açúcar cv. RB92579, sob regime de irrigação, no Semiárido brasileiro, visando a avaliar o desempenho desta cultura nas condições edafoclimáticas locais. Foram avaliados o acúmulo de biomassa seca e as suas respectivas partições, durante dois ciclos consecutivos de cana-de-açúcar (soca e ressoca), além dos indicadores de rendimento e de qualidade da cultura ao final do período experimental. Em ambos os ciclos, foram realizadas dez coletas de amostras para se determinar a biomassa seca de seis componentes estruturais dos perfilhos (folhas verdes, bainhas, parte emergente, pseudocolmos, folhas e bainhas mortas e colmo). A cana-de-açúcar apresentou elevado acúmulo de biomassa seca da parte aérea da planta, com valor médio de $6.493 \mathrm{~g} \mathrm{~m}^{-2}$ para os dois ciclos de cultivo. No início do seu crescimento, os fotoassimilados foram destinados prioritariamente às folhas verdes, bainhas, parte emergente e pseudocolmos. Em fase posterior de crescimento, os fotoassimilados passaram a ser utilizados na formação dos colmos. Os resultados obtidos foram muito semelhantes para os ciclos de soca e ressoca. Os modelos ajustados para descrever a evolução da biomassa seca e de suas respectivas partições apresentaram ajustes satisfatórios, em função dos dias após o corte. $\mathrm{O}$ elevado rendimento industrial $\left(133,88 \pm 40,84 \mathrm{t} \mathrm{ha}^{-1}\right)$ e a alta concentração de sacarose por biomassa seca $\left(0,34 \pm 0,10 \mathrm{~g} \mathrm{~g}^{-1}\right)$ proporcionaram valores elevados de produção de açúcar $\left(17,75 \pm 4,44 \mathrm{tha}^{-1}\right)$ e de álcool $\left(12,73 \pm 3,23 \mathrm{t} \mathrm{ha}^{-1}\right)$, indicando alto desempenho produtivo da RB92579 para a região.
\end{abstract}

Palavras-chave: análise de crescimento, bioenergia, Submédio do Vale do São Francisco.

\section{ABSTRACT}

\section{Accumulated dry biomass, partitioning and industrial yield of irrigated sugarcane in the Brazilian Semi-Arid}

The objective of this work was to evaluate growth and yield parameters of sugar cane cv. RB92579 under irrigation in the Brazilian semi-arid to evaluate performance of that crop in the local conditions of climate and soil. It was evaluated the accumulation of dry biomass and its respective partitions during two consecutive sugarcane ratoon cycles, as well as the indicators of industrial yield and crop quality at the end of the experimental period. Ten samples collections were carried out in both sugarcane cycles to determine the dry biomass of six structural

Recebido para publicação em 08/04/2013 e aprovado em 08/10/2013.

${ }^{1}$ Parte da tese de doutorado do primeiro autor, apresentada ao Programa de Pós-graduação em Meteorologia Agrícola, DEA/UFV, financiada pela Embrapa Semiárido / Agrovale / UFV / $\mathrm{CNPq}$

${ }^{2}$ Engenheiro-Agrônomo, Doutor. Unidade Acadêmica de Serra Talhada, Universidade Federal Rural de Pernambuco, Fazenda Saco, s/n, 56900-000, Serra Talhada, Pernambuco, Brasil. thieres_freire@yahoo.com.br(autor correspondente).

${ }^{3}$ Engenheiro-Agrônoma, Doutora. Empresa Brasileira de Pesquisa Agropecuária, Embrapa Semiárido, BR 428, Km 152, Zona Rural, CP 23, 56310-000, Petrolina, Pernambuco, Brasil. magna@cpatsa.embrapa.br

${ }^{4}$ Engenheiro Agrícola, Doutor. Departamento de Engenharia Agrícola, Universidade Federal de Viçosa, Campus Viçosa, Avenida Peter Henry Holfs s/n, CEP 36570-000, Viçosa, Minas Gerais, Brasil. zolnier@ufv.br

${ }^{5}$ Bióloga, Mestre. Unidade Acadêmica de Serra Talhada, Universidade Federal Rural de Pernambuco, Fazenda Saco, s/n, 56900-000, Serra Talhada, Pernambuco, Brasil. sanddrabastos@yahoo.com.br 
components of tillers (green leaves, sheaths, immature leaves and sheaths, pseudo-stem, dead leaves and sheaths and stems). The sugarcane presented high accumulation of dry biomass with a mean value of $6,493 \mathrm{~g} \mathrm{~m}^{-2}$ for the two cultivation cycles. At the beginning of the growing period, the photoassimilates are mainly destined to the green leaves, sheaths, immature leaves and pseudostem. Afterwards, the photoassimilates are used in the formation of stems and leaf enlargement. The results achieved in this work were very similar for both ratoon cycles. The fitted models used to describe the evolution of dry biomass and their respective partitions presented good correlation as a function of the days after cutting. The high industrial yield $\left(133.88 \pm 40.84 \mathrm{t} \mathrm{ha}^{-1}\right)$ and the high content of sucrose on the stem per dry biomass $\left(0.34 \pm 0.10 \mathrm{~g} \mathrm{~g}^{-1}\right)$ provided elevated values of sugar $\left(17.75 \pm 4.44 \mathrm{t} \mathrm{ha}^{-1}\right)$ and alcohol $\left(12.73 \pm 3.23 \mathrm{t} \mathrm{ha}^{-1}\right)$ production, indicating high productive performance of the RB92579 for the region.

Key words: bioenergy, growth analysis, lower middle of São Francisco Valley.

\section{INTRODUÇÃO}

A cana-de-açúcar é uma cultura bem adaptada às condições tropicais e subtropicais, caracterizadas por alta disponibilidade de água e de radiação luminosa. A planta apresenta grande capacidade de armazenamento de sacarose nos tecidos dos colmos, o que a torna de extrema importância comercial (Tejera et al., 2007). Porém, como ocorre com outras culturas agrícolas, o seu sistema de produção pode ser afetado por fatores relacionados com a planta (cultivares, tipo de ciclo), com o solo (propriedades físicas, fertilidade), com as práticas culturais (época de plantio, densidade de plantio, rotação de cultura) e, especialmente, com o clima (temperatura, radiação, precipitação) (Bell \& Garside, 2005; Park et al., 2005).

Os componentes estruturais da cana-de-açúcar (biomassa dos colmos e sacarose) condicionam a produção de açúcar ou de álcool, sendo que o incremento de um deles pode aumentar o rendimento final da cultura. Além disso, estas informações são utilizadas em modelos de simulação do crescimento e do rendimento dessa espécie (Singels et al., 2005). Para avaliar essa contribuição, análises do crescimento da cana-de-açúcar, em termos de produção de biomassa e de suas partições, podem ser de grande importância para melhorar o entendimento desses mecanismos em resposta às condições ambientais (Tejera et al., 2007).

Na cana-de-açúcar, quando não se verifica a intensificação do acúmulo de biomassa da parte aérea, uma partição mais favorável para os colmos pode promover maior conversão em sacarose, em decorrência do aumento de sólidos solúveis e do processo de desidratação. A redução da biomassa seca dos colmos, no entanto, não pode ser superior a $10 \%$, porque a concentração de sacarose varia com a partição de biomassa para os colmos, a qual por sua vez, é alterada em função do rendimento da cultura (Robertson \& Donaldson, 1998). Robertson et al. (1996) citam que a partição máxima de variedades de cana-deaçúcar com boa produtividade $\left(>6.000 \mathrm{~g} \mathrm{~m}^{-2}\right)$ é de 0,80 , para as condições edafoclimáticas da Austrália, sugerindo, também, valores entre 0,66 a 0,80, de acordo com o rendimento do cultivar. Evensen et al. (1997) relatam que, para as variedades cultivadas no Havaí, os valores de partição para os colmos situam-se em torno de 0,66 , quando a biomassa é superior a $5.000 \mathrm{~g} \mathrm{~m}^{-2}$. Segundo Inman-Bamber et al. (2002), os valores máximos de partição para os colmos devem ser da ordem de 0,85 , independentemente da variedade e do regime hídrico, para produtividades superiores a $6.000 \mathrm{~g} \mathrm{~m}^{-2}$, em condições tropicais. No entanto, segundo os mesmos autores, a colheita da cultura pode ser realizada quando o rendimento atingir pelo menos $4.000 \mathrm{~g} \mathrm{~m}^{-2}$, em condições subtropicais, e $6.000 \mathrm{~g} \mathrm{~m}^{-2}$, em condições tropicais. Como consequência, em termos de concentração de sacarose, os valores podem variar entre 0,35 e 0,50 $\mathrm{g}$ de sacarose por g de biomassa seca. Já Muchow et al. (1996) observaram que a concentração de sacarose em variedades bastante produtivas, nas condições climáticas da Austrália, independentemente do ciclo, atingiu valores máximos de $0,48 \mathrm{~g} \mathrm{~g}^{-1}$.

Essas informações são inexistentes para a cultura da cana-de-açúcar irrigada, nas condições edafoclimáticas do Submédio Vale do São Francisco, Brasil. Assim, o objetivo deste trabalho foi avaliar o acúmulo de biomassa seca e as suas partições, durante o período experimental, bem como, o desempenho industrial da cultivar RB92579 ao final do ciclo.

\section{MATERIAL E MÉTODOS}

\section{Época e condições edafoclimáticas}

Este estudo foi conduzido entre os meses de junho/ 2007 e junho/2008, com a cana-de-açúcar (Saccharum officinarum ssp.), cultivar RB92579, durante o ciclo de cana-soca, e de junho/2008 a julho/2009, durante o ciclo de ressoca. As condições meteorológicas ao longo do período são mostradas na Figura 1. O solo da área experimental é pertencente à classe dos Vertissolos.

Rev. Ceres, Viçosa, v. 61, n.5, p. 686-696, set/out, 2014 


\section{Local experimental}

$\mathrm{O}$ experimento foi conduzido em um talhão de canade-açúcar, pertencente à Empresa Agroindústrias do Vale do São Francisco (Agrovale), localizada no município de Juazeiro, Bahia, no Submédio do Vale do São Francisco, Semiárido do Nordeste brasileiro. O cultivo foi realizado, numa área de 12,17 ha, na direção leste-oeste, em fileiras com espaçamento de $1,5 \mathrm{~m}$.

\section{Tratos culturais}

Antes da implantação da cultura, que ocorreu em fevereiro de 2006, foi realizada uma adubação inicial com base nas análises de solo, sendo aplicados $112 \mathrm{~kg} \mathrm{ha}^{-1} \mathrm{de}$ $\mathrm{P}_{2} \mathrm{O}_{5}, 2,8 \mathrm{~kg}$ de $\mathrm{Cu}$ e de $\mathrm{Zn}$ e $1,2 \mathrm{~kg}$ de B, do formulado 29-08-00, enquanto, ao longo do ciclo de cana planta, adicionaram-se mais $125 \mathrm{~kg} \mathrm{ha}^{-1} \mathrm{de} \mathrm{N}$, na forma de ureia, e $135 \mathrm{~kg} \mathrm{ha}^{-1} \mathrm{de}_{2} \mathrm{O}$, na forma de cloreto de potássio.

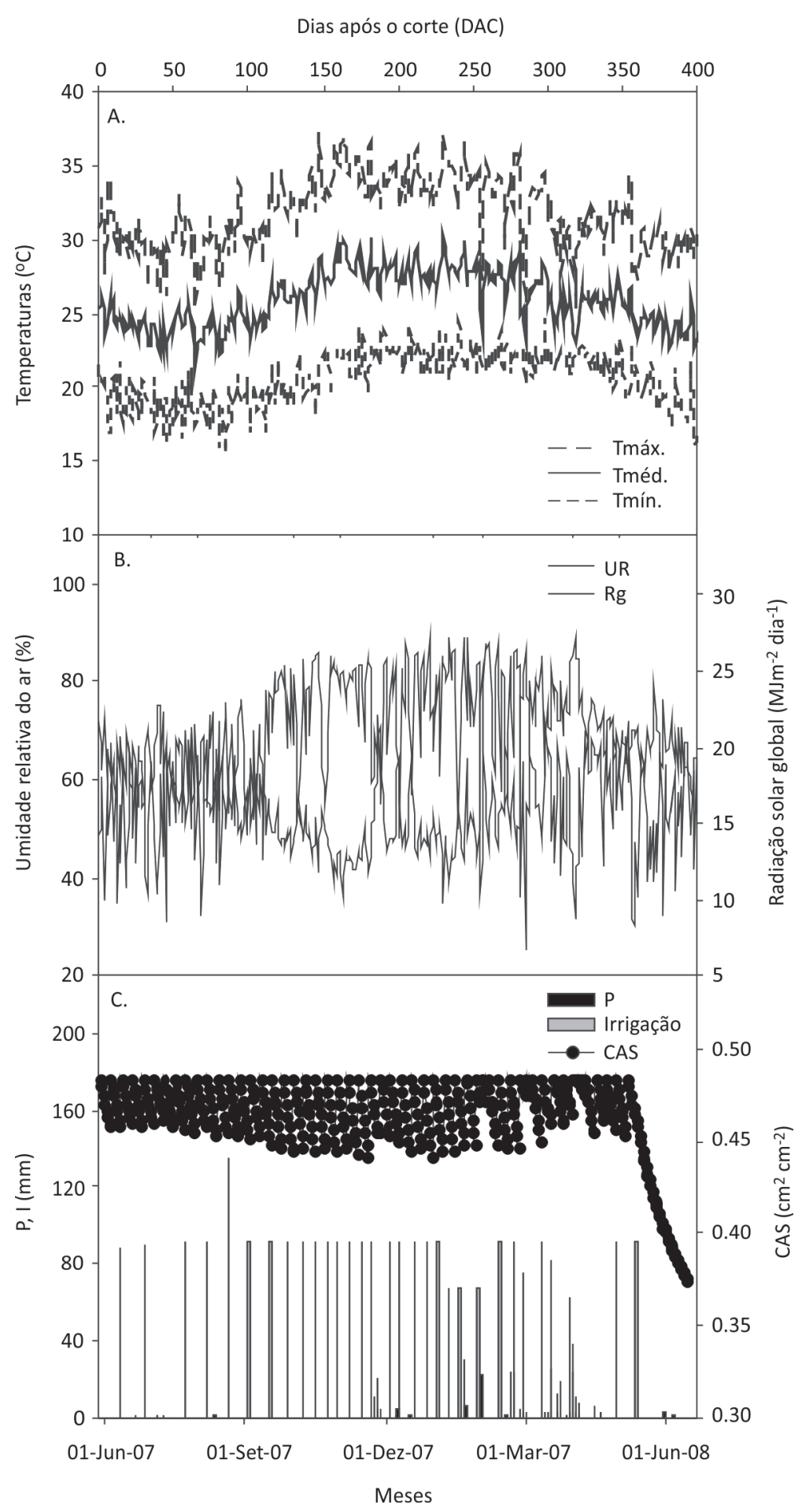

Figura 1. Variáveis meteorológicas e conteúdo de água no solo (CAS) medidos durante o período de cultivo da cana-de-açúcar irrigada, cultivar RB92579, no ciclo de cana-soca, sob condições climáticas do Semiárido brasileiro. 
Por sua vez, após a primeira colheita, quando se caracterizou o início do período experimental, ciclo de canasoca, foram aplicados, ao longo do tempo, $157,5 \mathrm{~kg} \mathrm{ha}^{-1}$ e $0,5 \mathrm{~L} \mathrm{ha}^{-1}$ de ureia e Ajipower, respectivamente, com base em análises de solo e foliar. Para o controle de ervas daninhas, foram aplicados $1 \mathrm{~L} \mathrm{ha}^{-1}$ de 2,4 D Amina e Glifosato. No ciclo subsequente (ressoca), foram aplicados $158,7 \mathrm{~kg} \mathrm{ha}^{-1}$ de $\mathrm{N}$ e 43,8 ha-1 de $\mathrm{P}$, a partir da fórmula 29-08-00, e de herbicidas à base de Diuron + Hexazinone, 2,4 D Amina e MSMA, nas quantidades de 2,48 kg ha-1 $472 \mathrm{ml} \mathrm{ha}^{-1}$ e 2,205 $\mathrm{L} \mathrm{ha}^{-1}$, respectivamente. Durante os dois ciclos, a irrigação foi realizada superficialmente, em sulcos, utilizando-se sistema de condução em tubos janelados, tendo os eventos sido realizadas com base no conteúdo de água no solo, obtido por meio de uma sonda FDR (Frequency Domain Reflectometer) modelo PR2/6 (Delta-T Devices Ltd., Burwell, Cambridge, UK) e a partir dos valores de evapotranspiração de referência (ETo). A irrigação foi realizada sempre que o conteúdo de água no solo atingisse $50 \%$ da capacidade de campo. Maiores detalhes da condução da área experimental podem ser obtidos em Silva (2009).

\section{Medidas de biomassa}

A biomassa seca da cana-de-açúcar foi obtida para seis componentes estruturais dos perfilhos, bem como para a parte aérea total, durante o ciclo de soca, utilizando-se uma metodologia proposta por Muchow et al. (1993), que tem sido bastante aplicada em estudos para esta cultura (Robertson et al., 1996; Inman-Bamber \& Mcglinchey, 2003; Inman-Bamber, 2004). A partir dessa metodologia, amostragens de perfilhos foram realizadas, ao nível do solo, em três subáreas representativas do talhão experimental, onde se coletou um volume de biomassa em quatro e cinco metros lineares ao longo da fileira de cultivo, de cada subárea, totalizando entre 18 e $24 \mathrm{~m}^{2}$ de material analisados por campanha de amostragem. Este material foi pesado, separadamente, por subárea, de modo a se obter a biomassa fresca total por parcela (BF1 biomassa fresca da primeira coleta). Desse total, foram amostrados 15 perfilhos representativos, por subárea, que foram divididos nos seis componentes estruturais, caracterizando a segunda coleta na mesma campanha de amostragem (BF2). Os componentes estruturais dos perfilhos compreenderam as folhas verdes (FV), as bainhas (B), as folhas e bainhas mortas (FBM), o pseudocolmo (PC), o colmo (C) e a parte emergente (PE).

A soma da biomassa fresca dos componentes estruturais dos 15 perfilhos de cada subárea forneceu a biomassa fresca total da parte aérea. Na sequência, todo o correspondente a cada um dos componentes estruturais foi triturado, separadamente, em uma forrageira, da qual fo- ram retiradas subamostras, caracterizando a terceira coleta (BF3) na mesma amostragem. Essas subamostras foram colocadas em recipientes de alumínio, pesadas em balança de precisão de 0,001 g (modelo MARK 210A, Bel Engineering, Monza-MI, Itália) e, posteriormente, secadas em estufa, com ventilação forçada e temperatura entre 65 e $70{ }^{\circ} \mathrm{C}$, até atingir massa constante. Após a secagem, determinou-se a biomassa seca da terceira coleta (BS3).

Os valores de biomassa seca foram expressos em gramas por metro quadrado. Essas informações foram obtidas, inicialmente, a partir da extrapolação dos dados das coletas do material triturado para o cálculo da biomassa seca dos 15 perfilhos de cada subárea (BS2 = BS3 x BF2 / BF3). Em seguida, calculou-se a fração que correspondia ( $\mathrm{fx}$, em que $\mathrm{x}=\mathrm{BFFV}, \mathrm{BFB}$, BFFBM, BFPC, BFC e BFPE) a cada componente estrutural dos perfilhos da biomassa fresca total da parte aérea (BFTPA), ou seja, $\mathrm{fx}=\mathrm{x} / \mathrm{BFTPA}$. Os valores de fx, BS2, BF3 e BF2 permitiram estimar a biomassa seca de cada componente estrutural dos perfilhos referente às coletas por subárea (BS1), ou seja, BS1 = fx.BS2.BF3 / BF2.

Diferentemente do ciclo de soca, no período de ressoca, os colmos representativos foram coletados diretamente nas subáreas de amostragem, em vez de se coletar um volume de quatro a cinco metros lineares ao longo da fileira de cultivo. Nesse ciclo, foram coletadas 4 plantas por subárea, totalizando 12 por amostragem. Para as demais etapas de obtenção da biomassa seca, foi adotado procedimento semelhante ao do ciclo anterior.

\section{Índices tecnológicos da cultura}

Ao final de ambos os ciclos da cana-de-açúcar, foram obtidos os seguintes índices tecnológicos, no laboratório de Análise Química, da Empresa Agroindústrias do Vale do São Francisco S.A. - Agrovale: sólidos solúveis totais, teor de fibra, teor de sacarose do caldo, pureza, percentagem bruta de açúcar, açúcares redutores livres, açúcares redutores totais e umidade. Com base nesses índices tecnológicos, foram calculados os rendimentos de açúcar e de álcool, conforme metodologia proposta por Caldas (1998), bem como o conteúdo de sacarose na biomassa seca dos colmos.

\section{Dados analisados}

A partir da relação entre os valores de biomassa seca de BSFV, BSB, BSFBM, BSPC, BSC e BSPE e de BSTPA de ambos os ciclos, foram obtidas as partições de biomassa para as partes estruturais da planta (pBSFV, pBSB, pBSFBM, pBSPC, pBSC e pBSPE), ao longo do tempo. Ambos os dados, de biomassa e das partições, foram submetidos a uma análise de regressão, em função dos valores de dias após o corte (DAP), com o programa 
SigmaPlot ${ }^{\circledR} 11$ (Systat Software Inc.). Foram usados os modelos Gaussiano $\left(y=a \cdot \exp \left(-0,5 .((x-b) / c)^{2}\right)\right)$, Sigmoidal $(y=a /(1+\exp (-(x-b) / c)))$ e Log Normal $(y=a \cdot \exp (-0,5 .(\ln (x /$ b)/c $\left.)^{2}\right)$ ) para os dados de biomassa, enquanto, para os dados das partições, adicionalmente se usou o modelo exponencial $(\mathrm{y}=\mathrm{a} \cdot \exp (-\mathrm{b} \cdot \mathrm{DAC})$, em que y é a variável dependente (biomassa e as suas respectivas partições); "a", "b" e "c" são os coeficientes dos modelos e, "x" a variável independente (DAC). As significâncias dos modelos e dos parâmetros pertinentes foram avaliadas com base no testes F e t de Student, respectivamente, considerando-se válidos desde que a $\mathrm{p}<0,05$. A análise dos ajustes dos modelos foi feita usando-se o coeficiente de determinação ajustado $\left(\mathrm{R}_{\text {Adj.), }}^{2}\right.$ que assume o número de colmos usado em cada regressão. Finalmente, utilizandose os dados de biomassa e dos índices tecnológicos, foram avaliados o desempenho industrial da cana-de-açúcar, por meio do TCH (toneladas de colmos por hectare), rendimento bruto de açúcar (RBAÇ), rendimento bruto de álcool (RBAL), concentração de sacarose nos colmos, em bases fresca $\left(\mathrm{CSC}_{\mathrm{bf}}\right)$ e seca $\left(\mathrm{CSC}_{\mathrm{bs}}\right)$.

\section{RESULTADOS E DISCUSSÃO}

A biomassa seca total da parte aérea (BSTPA) foi ligeiramente superior, para o ciclo de soca (385 dias) quando comparada à da ressoca (381 dias), resultando em valor médio de $6.493 \pm 227 \mathrm{~g} \mathrm{~m}^{-2}$ (Figura 2). Este rendimento é semelhante ao observado por Inman-Bamber et al. (2002), para as variedades Q96 e Q117, cultivadas sob diferentes épocas, num ciclo de cana-soca irrigado, com duração de 12 meses, cujos valores variaram de 5.000 a $6.800 \mathrm{~g} \mathrm{~m}^{-2}$. Ferreira Júnior et al. (2008), para as condições irrigadas do município de Rio Largo - AL, em ciclo de cana-soca de 13 meses, obtiveram uma biomassa seca acumulada de $6.600 \mathrm{~g} \mathrm{~m}^{-2}$. Contudo, nas condições de sequeiro no Estado de São Paulo, Almeida et al. (2008) verificaram valores de $3.500 \mathrm{~g} \mathrm{~m}^{-2}$ para a variedade RB92579. Estes resultados mostram que a maior eficiência produtiva da cana-de-açúcar, nas condições do Submédio do Vale do São Francisco, provavelmente esteve associada aos aspectos varietal e ao sistema de produção irrigado.

Dentro de cada ciclo, percebe-se que as variações das magnitudes da BSFV, BSB, BSPE e BSPC são semelhantes entre si, atingindo picos em relação às suas biomassas e redução ao longo do tempo, quanto às suas partições (Figuras 3 e 4). O mesmo pode ser afirmado em relação à similaridade entre os dois ciclos. Para ambos (Figura 3A), verificou-se que, no início do ciclo $(<100$ DAC), a BSFV era ascendente, representando, dentre os demais componentes estruturais, a maior fração, quanto à BSTPA, muito similar para ambos os ciclos (Figura 4A). Entretanto, a biomassa acumulada para as folhas durante o ciclo de soca foi superior à da ressoca. As partições registradas nos dois ciclos apresentam valores inferiores aos observados por Robertson et al. (1996), durante o mesmo período, que constataram valores de até 0,62 para a pBSFV. A partir desse momento (entre os 100 e $250 \mathrm{DAC}$ ), observou-se um crescimento acentuado da BSFV, quando os valores de temperatura do ar e de radiação solar global eram elevados (Figura $1 \mathrm{~A}$ e 1B). Contudo, notou-se que a fração destinada à BSFV (pBSFV) reduziu-se, em relação à destinada a BSTPA. No período subsequente (> 250 DAC), constatou-se uma diminuição da BSFV, resultando, ao final do ciclo, em menores valores de BSFV e pBSFV, com valor médio para a cultura na ordem de $229 \pm 20 \mathrm{~g} \mathrm{~m}^{-2}$ e de

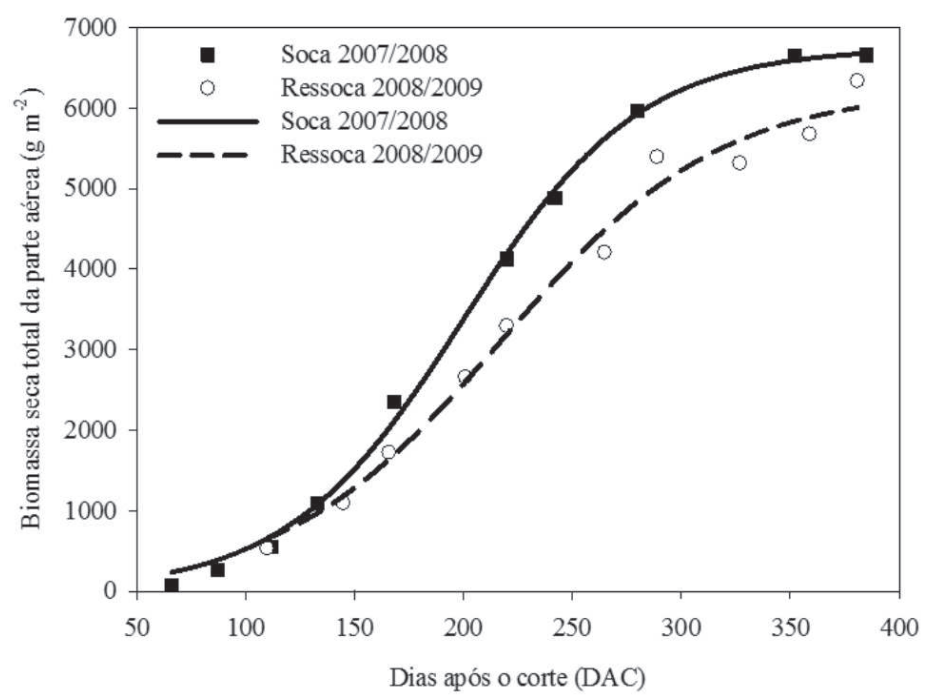

Figura 2. Biomassa seca total da parte aérea da cana-de-açúcar irrigada, variedade RB92579, durante os ciclos de soca (2007/2008) e ressoca (2008/2009), sob condições climáticas do Semiárido brasileiro. 


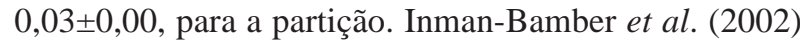
verificaram, ao final do ciclo, para as variedades Q36 e Q117, que a pBSFV esteve em torno de 0,1 , quando a BSTPA foi superior a $4000 \mathrm{~g} \mathrm{~m}^{-2}$, e 0,40 quando a BSTPA foi inferior a $4000 \mathrm{~g} \mathrm{~m}^{-2}$.

No que concerne à biomassa seca das folhas e das bainhas mortas (BSFBM), verificou-se tendência crescente (Figura 3B), sendo superior na ressoca. No início do ciclo, quando a cultura ainda apresentava um IAF baixo, observou-se que a BSFBM foi apenas expressiva a partir dos 100 DAC. Nesse momento, a pBSFBM era baixa (Figura 4B). Com o decorrer do ciclo, o surgimento de novas folhas promoveu um aumento da quantidade de BSFBM e, com isso, um aumento da pBSFBM. Ao final do ciclo, os seus respectivos valores médios foram iguais a $1.080 \pm 99 \mathrm{~g} \mathrm{~m}^{-2}$ e 0,16 $\pm 0,01$. Inman-Bamber et al. (2002) constataram valores de pBSFBM entre 0,18 e 0,22 , ao final do ciclo, respectivamente para as variedades NCo376 e N12. Sob condições de sequeiro, com precipitação pluviométrica em torno de $669 \mathrm{~mm}$, os valores de pBSFBM tenderam a aumentar, atingindo 0,36 para a variedade N16 e 0,31 para a NCo376, como respostas às diferenças dos mecanismos de tolerância à seca.

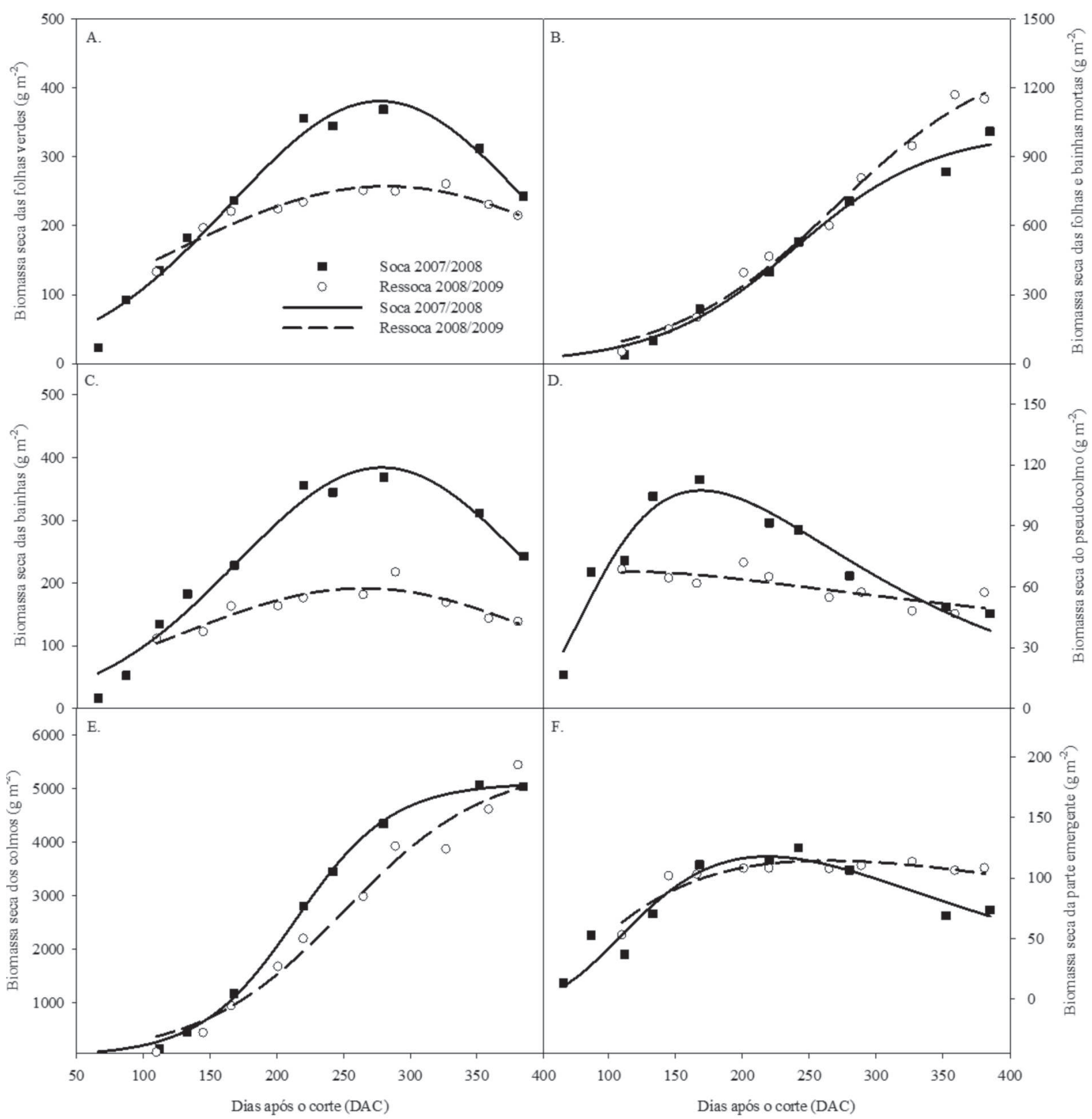

Figura 3. Biomassa seca acumulada das folhas verdes (A), bainhas (B), parte emergente (C), pseudocolmo (D), folhas e bainhas mortas (E) e colmo (F) para a cana-de-açúcar irrigada, variedade RB92579, durante os ciclos de soca (2007/2008) e ressoca (2008/ 2009), sob condições climáticas do Semiárido brasileiro. 
A biomassa seca acumulada para as bainhas (BSB) (Figura 3C) obteve um comportamento similar ao obtido para a BSFV. Contudo, a fração destinada a BSB (pBSB) foi inferior à da BSFV (Figura 4A), antes dos 100 DAC. A partir desse período, o valor de BSB mostrou-se muito próximo ao da BSFV. Ao final do ciclo, a BSB foi de 191 \pm 74 $\mathrm{g} \mathrm{m}^{-2}$, entretanto, a pBSB $(0,03 \pm 0,01)$ foi igual a pBSFV.

Para a biomassa seca acumulada do pseudocolmo (BSPC) (Figura 3D), o período de máxima magnitude (entre os 150 e 250 DAC) coincide com a fase de maior taxa de elongação média dos colmos, de grande importância para o rendimento final desta cultura. A partir deste momento, verificou-se uma redução dos valores de BSPC, ao final do ciclo $\left(57 \pm 14 \mathrm{~g} \mathrm{~m}^{-2}\right)$. Observou-se, também, que, entre 150 e $250 \mathrm{DAC}$, ocorreu a maior emissão de folhas pela cultura. Além disso, semelhantemente aos da BSFV e BSB (Figura 4D), os valores durante a ressoca foram menores, enquanto as partições foram bastante similares $(0,01 \pm 0,00)$ (Figura 4D).

A maior partição de biomassa ocorreu para os colmos (Figura 4E). Pode-se perceber uma simetria entre os valores de pBSC e de pBSPC (Figura 4D), ou seja, quando um aumenta o outro se reduz, de modo que isso fortalece a teoria da relação fonte-dreno entre essas duas es-

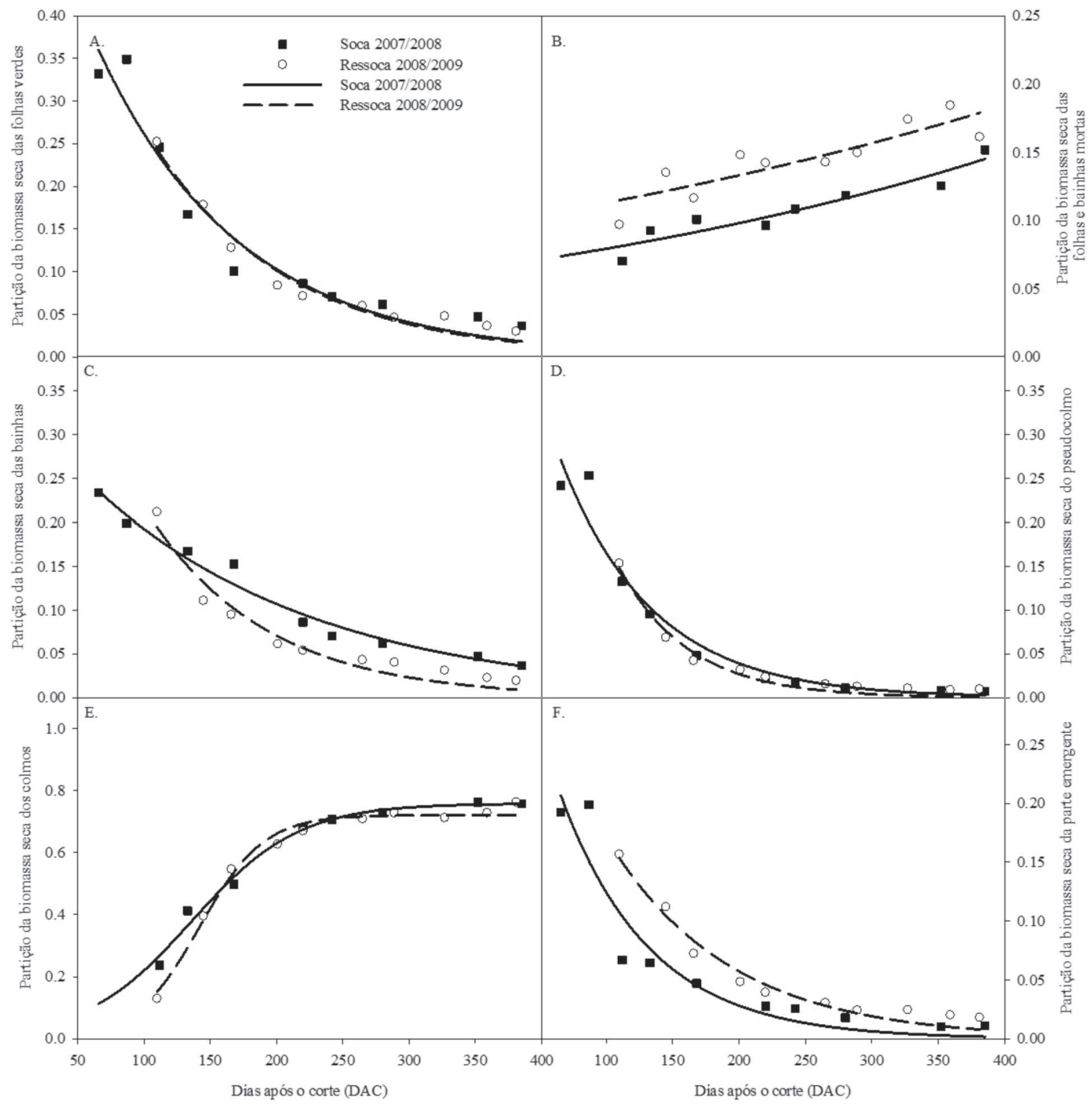

Figura 4. Partições da biomassa seca das partes estruturais (folhas verdes, A; bainhas, B; parte emergente, C; pseudocolmo, D; folhas e bainhas mortas, E; e, colmo, F). Os valores calculados têm como referência a biomassa seca da parte área da cana-de-açúcar irrigada, variedade RB92579, durante os ciclos de soca (2007/2008) e ressoca (2008/2009), sob condições climáticas do Semiárido brasileiro. 
truturas (Liu \& Bull, 2001), comportamento esse semelhante entre os ciclos produtivos. Antes dos 100 DAC, não foi verificada formação pronunciada de BSC, de modo que toda a biomassa era destinada a BSPC (Figura 3D). Entretanto, a partir daí, observou-se que a BSC já era maior, tendendo a aumentar rapidamente. Fato semelhante foi observado com a elongação dos colmos, resultando uma produção da ordem de $4.835 \pm 286 \mathrm{~g} \mathrm{~m}^{-2}$, ao final do ciclo, decorrente de uma maior partição para os colmos, que foi em torno de $0,76 \pm 0,00$. InmanBamber et al. (2002) constataram, nas condições climáticas da África do Sul, valores médios de pBSC em torno de 0,85, para várias variedades e locais de cultivo. Para um ano mais seco ( 669 mm), estes autores notaram que a BSC variou entre 2000 e $3000 \mathrm{~g} \mathrm{~m}^{-2}$, enquanto, para um ano mais chuvoso $(\sim 1098 \mathrm{~mm})$, a cultura apresentou valores de BSC variando entre 3.000 a 4.000 $\mathrm{g} \mathrm{m}^{-2}$. Para as condições climáticas da Austrália, quando o rendimento foi de 5.000 a $6.000 \mathrm{~g} \mathrm{~m}^{-2}$, os valores médios de pBSC oscilaram em torno de 0,80 .

A biomassa seca acumulada da parte emergente (BSPE) (Figura 3F) e sua partição (pBSPE) em relação à BSTPA (Figura 5F) apresentaram comportamento semelhante ao da BSPC (Figuras 3D e 4D), com os picos ocorrendo entre os 150 e 250 DAC. Ao final do ciclo, os valores de BSPE $\left(101 \pm 39 \mathrm{~g} \mathrm{~m}^{-2}\right)$ e de pBSPE $(0,01 \pm 0,00)$ foram reduzidos, sendo superiores no ciclo de ressoca. Inman-Bamber et al. (2002) verificaram valores máximos de 0,05 no início do ciclo. No entanto, seus valores tenderam a aproximar-se de zero ao final do ciclo. Esses valores são bem inferiores aos verificados neste trabalho (Figura 4C).

A soma dos valores da BSPC e da BSPE resulta num componente denominado, internacionalmente, "cabbage". No experimento deste estudo, seus valores tenderam a se reduzir até o final do ciclo, atingindo $158,28 \mathrm{~g} \mathrm{~m}^{-2}$, em ter-

Tabela 1. Parâmetros dos modelos exponencial $(\mathrm{Y}=\mathrm{a} \cdot \exp (\mathrm{b} \cdot \mathrm{DAC}))$, Gaussiano $\left(\mathrm{Y}=\mathrm{a} \cdot \exp \left(-0,5 \cdot((\mathrm{DAC}-\mathrm{b}) / \mathrm{c})^{2}\right)\right)$, Sigmoidal $(\mathrm{Y}=\mathrm{a} /$ $(1+\exp (-(\mathrm{DAC}-\mathrm{b}) / \mathrm{c})))$ e $\log \operatorname{Normal}\left(\mathrm{Y}=\mathrm{a} \cdot \exp \left(-0,5 .(\ln (\mathrm{DAC} / \mathrm{b}) / \mathrm{c})^{2}\right)\right)$ ajustados para a biomassa seca acumulada das partes estruturais e das suas respectivas partições da cana-de-açúcar irrigada, variedade RB92579, durante os ciclos de soca (2007/2008) e ressoca (2008/2009), sob condições climáticas do Semiárido brasileiro. A variável independente DAC refere-se ao número de dias transcorridos após o corte

\begin{tabular}{|c|c|c|c|c|c|c|}
\hline \multicolumn{7}{|c|}{ Biomassa seca acumulada } \\
\hline Ciclo & Componente estrutural (Y) & Modelo & a & b & c & $\mathbf{R}^{2}{ }_{\text {Adj }}$ \\
\hline \multirow{7}{*}{ Soca $(2007 / 2008)$} & Folhas & Gaussiano & 380,5704 & 277,4524 & 112,2953 & 0,97 \\
\hline & Bainhas e folhas mortas & Sigmoidal & 1009,8844 & 239,3421 & 51,6627 & 0,98 \\
\hline & Bainhas & Gaussiano & 384,0967 & 108,5710 & 278,7345 & 0,96 \\
\hline & Pseudocolmo & Log Normal & 107,4264 & 168,9519 & 0,5750 & 0,90 \\
\hline & Colmos & Sigmoidal & 5107,9660 & 214,3091 & 35,8965 & 0,99 \\
\hline & Parte emergente & Log Normal & 117,7152 & 218,3726 & 0,5438 & 0,86 \\
\hline & Parte aérea & Sigmoidal & 6743.9809 & 199.9846 & 40.3378 & 0,99 \\
\hline \multirow{7}{*}{ Ressoca (2008/2009) } & Folhas & Gaussiano & 257,2028 & 282,1307 & 166,9161 & 0,88 \\
\hline & Bainhas e folhas mortas & Sigmoidal & 1375,4790 & 270,2227 & 62,3468 & 0,99 \\
\hline & Bainhas & Gaussiano & 191,7905 & 264,6157 & 139,9126 & 0,77 \\
\hline & Pseudocolmo & Log Normal & 79,3399 & 121,6861 & 1,4511 & 0,53 \\
\hline & Colmos & Sigmoidal & 5448,0117 & 250,7857 & 53,7012 & 0,97 \\
\hline & Parte emergente & Log Normal & 134,1688 & 262,6394 & 0,9893 & 0,81 \\
\hline & Parte aérea & Sigmoidal & 6232.1129 & 217.6495 & 50.0445 & 0,99 \\
\hline \multicolumn{7}{|c|}{ Partições da biomassa seca acumulada } \\
\hline Ciclo & Componente estrutural (Y) & Modelo & a & b & c & $\mathbf{R}_{\text {Adj }}^{2}$ \\
\hline \multirow{6}{*}{ Soca $(2007 / 2008)$} & Folhas & Exponencial & 0,6648 & $-0,0093$ & - & 0,94 \\
\hline & Bainhas e folhas mortas & Exponencial & 0,0643 & 0,0021 & - & 0,88 \\
\hline & Bainhas & Exponencial & 0,3466 & $-0,0059$ & - & 0,97 \\
\hline & Pseudocolmo & Exponencial & 0,7008 & $-0,0144$ & - & 0,95 \\
\hline & Colmos & Sigmoidal & 0,7585 & 40,2896 & 136,0608 & 0,98 \\
\hline & Parte emergente & Exponencial & 0,5514 & $-0,0149$ & - & 0,89 \\
\hline \multirow{6}{*}{ Ressoca (2008/2009) } & Folhas & Exponencial & 0,7109 & $-0,0097$ & - & 0,97 \\
\hline & Bainhas e folhas mortas & Exponencial & 0,0961 & 0,0016 & - & 0,73 \\
\hline & Bainhas & Exponencial & 0,6686 & $-0,0112$ & - & 0,94 \\
\hline & Pseudocolmo & Exponencial & 1,2037 & $-0,0190$ & - & 0,97 \\
\hline & Colmos & Sigmoidal & 0,7212 & 24,2651 & 142,0609 & 0,98 \\
\hline & Parte emergente & Exponencial & 0,5140 & $-0,0110$ & - & 0,96 \\
\hline
\end{tabular}


mos de biomassa seca, e $0,02 \pm 0,01$ quanto à partição. Para variedades com produção de BSTPA acima de 5.000 $\mathrm{g} \mathrm{m}^{-2}$, Robertson et al. (1996) citam valores de "cabbage" em torno de 0,05, no final do ciclo. Inman-Bamber et al. (2002) informam valores de 0,10 para variedades de cana-de-açúcar com produção de BSTPA até $3.000 \mathrm{~g} \mathrm{~m}$ -

${ }^{2}$. Contudo, à medida que ocorre aumento da BSTPA (de 3.000 a $6.000 \mathrm{~g} \mathrm{~m}^{-2}$ ), maior quantidade de BSPC é convertida em rendimento final, de modo que os valores de "cabbage" podem-se reduzir para a faixa de 0,05 a 0,07 .

A biomassa seca total da parte área, subtraída da biomassa dos colmos, é conhecida como "palhada", que corresponde aos demais componentes da planta, a qual pode ser incorporada ao solo como matéria orgânica ou utilizada para a produção de energia em termoelétricas. No experimento deste estudo, verificou-se uma redução pronunciada da fração destinada à palhada, ao longo do tempo, em decorrência do aumento da BSC. Ao final do ciclo produtivo, assumindo-se os dois ciclos, a biomassa de palhada foi de $1658 \pm 59 \mathrm{~g} \mathrm{~m}^{-2}$, correspondendo a $0,24 \pm 0,00$ da BSTPA.

Os modelos ajustados para a biomassa acumulada e suas respectivas partições entre os componentes estruturais da planta podem ser observados na Tabela 1. Em termos de biomassa acumulada, verifica-se que os modelos apresentaram ótimos ajustes com o uso de dias após o corte (DAC) como variável independente, proporcionando altos valores para o coeficiente de determinação $\left(R_{2 A d j}\right)$, sobretudo para o ciclo de soca. O mesmo foi constatado para os modelos ajustados para as suas partições, com exceção daquele para a biomassa seca das folhas e bainhas mortas, para os quais o ajuste foi inferior $\left(\mathrm{R}_{2 \mathrm{Adj}}\right.$. $0,73)$, no ciclo de ressoca, o que está associada à grande dificuldade de seu monitoramento em campo.

Os valores dos índices tecnológicos e dos indicadores de rendimento da cultura demonstram que, de modo geral, as suas magnitudes tendem a se reduzir do ciclo de soca para o ciclo de ressoca (Tabela 2). Essa tendência tem sido citada por Dalri \& Cruz (2008). Todavia, os coeficientes de variação, entre os dois ciclos, são baixos $(<30,50 \%)$, sobretudo em relação aos índices tecnológicos $(<8,82 \%)$, indicando características intrínsecas à variedade RB92579. Em média, a $\mathrm{TCH}$ foi igual a $133,88 \pm 40,84 \mathrm{t} \mathrm{ha}^{-1}$, superior à do ciclo de soca e próxima à informada por Almeida et al. (2008), que observaram um rendimento de TCH de $136,22 \mathrm{t} \mathrm{ha}^{-1}$, para a mesma variedade (RB 92579), sob as condições irrigadas do Estado de São Paulo. Em termos de RBAÇ, verificou-se que o rendimento foi de $17,75 \pm 4,44 \mathrm{tha}^{-1}$, enquanto o RBAL foi de $12,73 \pm 3,23$ $\mathrm{m}^{3} \mathrm{ha}^{-1}$. Dantas Neto et al. (2006), trabalhando com a variedade SP 791011, submetida a várias lâminas de irrigação, sob as condições climáticas de Capim-PB,

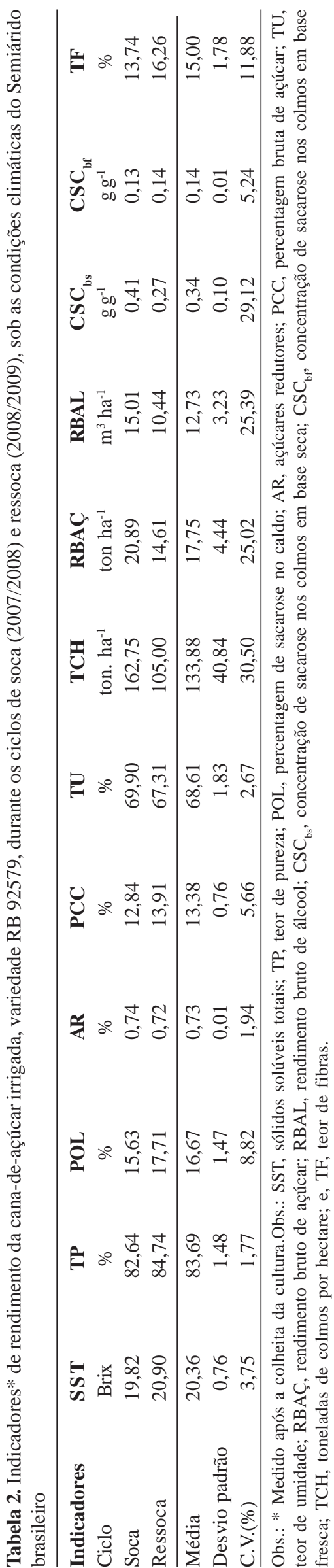

Rev. Ceres, Viçosa, v. 61, n.5, p. 686-696, set/out, 2014 
obtiveram valores de RBAÇ entre 9 e $12,58 \mathrm{t} \mathrm{ha}^{-1}$ e de RBAL entre 6,25 e 8,91 $\mathrm{m}^{3}$ ha $^{-1}$. Já Azevedo (2002), encontrou valor médio de RBAÇ da ordem de $14,71 \mathrm{t} \mathrm{ha}^{-1}$, para a cana-planta, enquanto Wiedenfeld (2000), trabalhando com a cana-de-açúcar sob três condições de irrigação $(95,85$ e $65 \%$ do limite crítico de água no solo para a cultura), constatou rendimentos de 13, 10 e 8 tha${ }^{1}$ de açúcar, respectivamente. Comparando-se estes dados aos obtidos neste estudo, é possível inferir que a cana-de-açúcar irrigada, sob as condições do Submédio do Vale do São Francisco, apresenta alta capacidade de conversão de biomassa dos colmos em sacarose, uma vez que apresentou alto rendimento industrial.

Por outro lado, o teor de fibras $(15,00 \pm 1,78 \%)$ foi comparável aos obtidos por diferentes autores, como, por exemplo, Carvalho et al. (2008), que citam valores de TF variando entre 13,91 e 13,32\% para a variedade SP 791011, dependendo dos níveis de água aplicados por irrigação (Tabela 2). Por outro lado, Silva (2002), avaliando a resposta da variedade SP 716949 em diferentes níveis de adubação, nos tabuleiros costeiros da Paraíba, encontrou valores de TF em torno de $14,20 \%$, enquanto Marques et al. (2007), analisando os índices tecnológicos de seis variedades de cana-de-açúcar (SP 801816, RB 845210, SP 813250, RB 855536, RB 867515 e RB 72454), verificaram valores de TF variando entre 12,02 e $13,83 \%$.

Em termos de concentração de açúcar nos colmos (TSC), observou-se que foi em torno de $0,34 \pm 0,10$ gramas de açúcar por gramas de matéria seca $\left(\mathrm{g} \mathrm{g}^{-1}\right)$, enquanto em base de biomassa fresca foi de $0,14 \pm 0,01 \mathrm{~g} \mathrm{~g}^{-1}$. Em termos de base seca, este valor é semelhante aos citados por Inman-Bamber et al. (2002), que variaram entre 0,35 e $0,50 \mathrm{~g} \mathrm{~g}^{-1}$, e inferiores aos valores obtidos por Robertson et al. (1999), para a variedade Q96 irrigada, sob as condições climáticas da Austrália, em que os valores variaram entre 0,47 e $0,53 \mathrm{~g} \mathrm{~g}^{-1}$.

\section{CONCLUSÃO}

A cana-de-açúcar RB92579 apresentou elevado acúmulo de biomassa sob as condições irrigadas do Submédio São Francisco, sendo que, inicialmente, os fotoassimilados são destinados aos componentes estruturais, que compreendem as folhas verdes, bainhas, parte emergente e pseudocolmos. Posteriormente, os fotoassimilados são direcionados prioritariamente para a formação dos colmos e, de maneira complementar, há aumento da biomassa seca das folhas e das bainhas mortas das plantas.

Os comportamentos do acúmulo de biomassa e de suas partições são bastante semelhantes para os ciclos de soca e ressoca.
A evolução da biomassa seca e de suas respectivas partições apresentou bons ajustes com a utilização de dias após o corte como variável independente.

O elevado rendimento agronômico da cultivar RB92579 resultou em alta concentração de sacarose por biomassa seca e, consequentemente, elevada produção de açúcar e álcool, sugerindo um ótimo desempenho industrial desta variedade nas condições edafoclimáticas do Semiárido brasileiro.

\section{REFERÊNCIAS}

Almeida AC dos S, Souza JL, Teodoro I, Barbosa GVS, Moura Filho G \& Ferreira Júnior RA (2008) Desenvolvimento vegetativo e produção de variedades de cana-de-açúcar em relação à disponibilidade hídrica e unidades térmicas. Ciência e Agrotecnologia, 32:1441-1448.

Azevedo H M (2002) Resposta da cana-de-açúcar a níveis de irrigação e de adubação de cobertura nos tabuleiros da Paraíba. Tese Doutorado. Campina Grande, Universidade Federal de Campina Grande. 112p.

Bell MJ \& Garside AL (2005) Shoot and stalk dynamics and the yield of sugarcane crops in tropical and subtropical Queensland, Australia. Field Crops Research, 92: 231-248.

Caldas C (1998) Manual de análises selecionadas para indústrias sucroalcooleiras. Maceió, Sindicato da Indústria e do Álcool do Estado de Alagoas. $438 \mathrm{p}$.

Carvalho CM de, Azevedo HM de, Dantas Neto J, Melo EP de, Silva CTS da \& Gomes Filho RR (2008) Resposta dos parâmetros tecnológicos da terceira folha de cana-de-açúcar submetida a diferentes níveis de irrigação. Revista Brasileira de Ciências Agrárias, 3:337-342.

Dantas Neto J, Figueiredo JL da C, Farias CH de A, Azevedo HM de \& Azevedo CAV de (2006) Resposta da cana-de-açúcar, primeira soca, a níveis de irrigação e adubação de cobertura. Revista Brasileira de Engenharia Agrícola e Ambiental, 10:283-288.

Dalri AB, Cruz RL (2008) Produtividade da cana-de-açúcar fertirrigada com N e K via gotejamento subsuperficial. Engenharia Agrícola, 28(3): 516-524

Evensen CI, Muchow RC, El-Swaify A. \& Osgood RV (1997) Yield accumulation in irrigated sugarcane. I. Effect of crop age and cultivar. Agronomy Journal, 89:638-646.

Ferreira EA, Aspiazú I, Galon L, Conçenço G, Silva AF, Santos FCMT, Ferreira FA, Barbosa MHP \& Silva AA (2008) Características fisiológicas de cinco cultivares de cana-de-açúcar. In: $9^{\circ}$ Congresso Nacional da Sociedade dos Técnicos Açucareiros e Alcooleiros do Brasil, Maceió. Anais, STAB. 598-601p.

Inman-Bamber NG (2004) Sugarcane water stress criteria for irrigation and drying off. Field Crops Research, 89:107-122.

Inman-Bamber NG \& Mcglinchey MG (2003) Crop coefficients and water-use estimates for sugarcane based on long-term bowen ratio energy balance measurements. Field Crops Research, Amsterdam, 83:125-138.

Inman-Bamber NG, Muchow RC \& Robertson MJ (2002) Dry partitioning of sugarcane in Australia and South Africa. Field Crops Research, 76:71-84.

Liu DL \& Bull TA (2001) Simulation of biomass and sugar accumulation in sugarcane using a process-based model. Ecological Modelling, 144:181-211.

Marques TA, Godinho AMM, Tiritan CS, Creste JE \& Marques MO (2007) Parâmetros biométricos e tecnológicos de cultivares de canade-açúcar para o Oeste Paulista. Stab, açúcar, álcool e subprodutos, 26:6-10, 2007. 
Muchow RC, Robertson MJ \& Wood AW (1996) Growth of sugarcane under high input conditions in tropical Australia. II. Sucrose accumulation and commercial yield. Field Crops Research, 48:27-36.

Muchow RC, Wood AW, Spillman MF, Robertson MJ \& Thomas MR (1993) Field techniques to quantify the yield determining processes in sugarcane. I. Methodology. In: $15^{\text {a }}$ Conference of Australian Society of Sugar Cane Technologists, Brisbane. Proceedings, CSIRO. p.336-343.

Park SE, Robertson M \& Inman-Bamber NG (2005) Decline in the growth of a sugarcane crop with age under high input conditions. Field Crops Research, 92:305-320.

Robertson MJ \& Donaldson RA (1998) Changes in the components of cane and sucrose yield in response to drying-off before harvest. Field Crops Research, 55:201-208.

Robertson MJ, Inman-Bamber NG, Muchow RC \& Wood AW (1999) Physiological analysis of early season deficit and sugarcane productivity. Field Crops Research, Amsterdan, 64:211-227.

Robertson MJ, Wood AW \& Muchow RC (1996) Growth of sugarcane under high input tropical conditions. I. Radiation use, biomass accumulation and partitioning. Field Crops Research, 48:11-25.
Silva AB da (2002) Resposta da cana-de-açúcar irrigada sob diferentes níveis de adubação. Dissertação Mestrado. Campina Grande, Universidade Federal de Campina Grande. 61p.

Silva TGF da (2009) Análise de crescimento, interação biosfera-atmosfera e eficiência do uso de água da cana-de-açúcar irrigada no Submédio do Vale do São Francisco. Tese Doutorado. Viçosa, UFV. $194 p$.

Singels A, Smit MA, Redshaw KA \& Donaldson RA (2005) The effect of crop start date, crop class and cultivar on sugarcane canopy development and radiation interception. Field Crops Research, 92:249260

Tejera NA, Rodés R, Ortega E, Campos R \& Lluch C (2007) Comparative analysis of physiological characteristics and yield components in sugarcane cultivars. Field Crops Research, 102:64-72.

Wiedenfeld RP (2000) Water stress during different sugarcane growth periods on yield and response to $\mathrm{N}$ fertilizer. Agricultural Water Management, 43:173-182. 